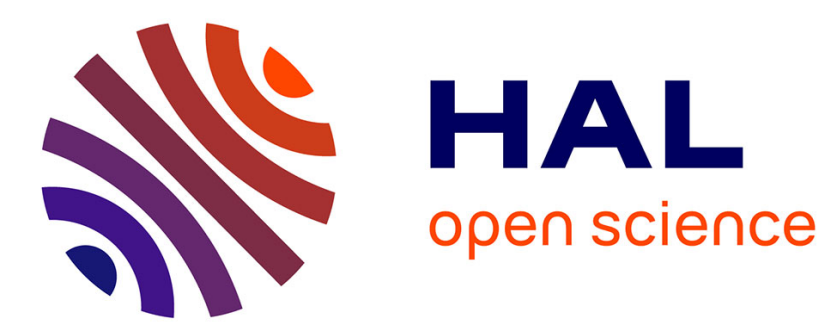

\title{
Multi-scale and multi-representation CAD models reconciliation for knowledge synthesis
}

Lionel Roucoules, Frédéric Demoly

\section{To cite this version:}

Lionel Roucoules, Frédéric Demoly. Multi-scale and multi-representation CAD models reconciliation for knowledge synthesis. CIRP Annals - Manufacturing Technology, 2020, 69 (1), pp.137-140. 10.1016/j.cirp.2020.04.089 . hal-03145093

\section{HAL Id: hal-03145093 \\ https://hal.science/hal-03145093}

Submitted on 4 Mar 2021

HAL is a multi-disciplinary open access archive for the deposit and dissemination of scientific research documents, whether they are published or not. The documents may come from teaching and research institutions in France or abroad, or from public or private research centers.
L'archive ouverte pluridisciplinaire HAL, est destinée au dépôt et à la diffusion de documents scientifiques de niveau recherche, publiés ou non, émanant des établissements d'enseignement et de recherche français ou étrangers, des laboratoires publics ou privés. 


\title{
Multi-scale and multi-representation CAD models reconciliation for knowledge synthesis
}

\author{
Lionel Roucoules $(2)^{\mathrm{a}, *}$, Frédéric Demoly ${ }^{\mathrm{b}}$ \\ ${ }^{a}$ Arts et Metiers Institute of Technology, LISPEN, HESAM Université, F-13617 Aix-en-Provence, France \\ ${ }^{\mathrm{b}}$ ICB UMR 6303 CNRS, Univ. Bourgogne Franche-Comté, UTBM, Belfort, France
}

A R T I C L E I N F O

\section{Keywords:}

Design method

Computer Aided Design (CAD)

Knowledge synthesis

\begin{abstract}
A B S T R A C T
Over the last decades, concurrent engineering and design for $\mathrm{X}$ approaches have introduced knowledgebased decision supports, analysis methods and feature-based modelling techniques to deliver designed solutions ready for specific lifecycle purposes. However, to cover the emerging knowledge synthesis issue in engineering design, the underlying 3D representations need to be better understood and chained. This paper aims at developing original multi-representation and multi-scale CAD models to integrate properly knowledge in their most suitable form. Reconciliation mechanisms can be set up to validate semantic continuity of the geometric models and to justify the knowledge structuring the design solutions space.
\end{abstract}

\section{Introduction}

Over the last six decades, computer-aided design (CAD) has received intensive attention from academia and industry. amongst the abundant research efforts, one can say that most of them have been derived from engineering design strategies [1]. The latter has shift from sequential to concurrent and integrated, and recently towards proactive and generative design. This has consequently led to the development of dedicated CAD approaches supporting underlying strategies such as organizational strategies (e.g. distributed modelling, collaborative modelling, multilevel modelling, etc.), lifecycle phase integration (e.g. manufacturing orientated, assembly orientated, etc.) with design for X methods, hierarchy strategies (namely bottom-up and top-down), knowledge reuse strategies (verification, parameter-based, feature-based, template-based, etc.), just to name a few. CAD models are now well democratized and considered as a ubiquitous information technology able to address multiple stakeholders' purposes [2].

In engineering design, CAD models have required the support of product lifecycle management (PLM) systems as digital backbone to provide the right information at the right person at the right time in a suitable format. In such ecosystems, CAD models go along with metamodels for data structuring, mapping and dissemination [3]. Nevertheless, these strong sets of adopted approaches and systems - once faced towards lifecycle knowledge integration - led to both relevant issues: (i) complexity in CAD models interpretation with multiple allocated constraints and knowledge from downstream processes and disciplines, and (ii) semantic continuity over the multiple heterogeneous stakeholders' models including the geometric model along the product lifecycle. Therefore, promoting trade-offs in engineering design and then knowledge synthesis for improving decision-making, demands to overcome the aforementioned barriers. In such a context, the paper's objective is

\footnotetext{
* Corresponding author

E-mail address: Lionel.Roucoules@ensam.eu (L. Roucoules).
}

to enrich design method to have lifecycle knowledge be instantiated in a proactive manner and allocated at the right time on the right geometric objects during the conceptual, embodiment and detailed design stages. Adopting this strategy in the design process contributes to the emergence by 'least commitment' of the product geometry, which is thus the pure result of Lifecycle knowledge synthesis [2].

The article highlights in Section 2 the scientific questions with significant reported works and presents the hypotheses of the research work. Afterwards, in Section 3, an original flow-based interface model built toward an emerging CAD modelling technique is proposed. Such model aims at ensuring the semantic continuity between design intents (i.e. lifecycle knowledge) and geometric objects; then enabling the reconciliation of multi-scale and multi-representation of CAD models.

\section{Research questions and hypotheses}

The paper discusses - with the support of key published research works - two research hypotheses for which it provides original solutions.

\subsection{Digital continuity between stakeholders' design intents and geometric models for multi-scale knowledge synthesis}

Current design practices focus too quickly on the definition of a single solution. As a result, all stakeholders of the product development process (PDP) achieve analysis and make decisions on a unique and central CAD representation. This arbitrarily reduces the design solutions space and does not raise stakeholders' awareness on the relevant knowledge to be considered [2]. In a 'least commitment' approach, the lifecycle knowledge synthesis becomes vital in order to push the emergence of the geometric model gradually. Moreover, this allows managing the influence of knowledge and decisions' evolution of each of the stakeholders on the final geometric model $[4,5]$.

Over the past decades, researchers have addressed part of this issue by developing knowledge-based approaches that rely on meta- 
models. The latter actually enables the concurrent modelling of stakeholders' design intents [3], however, the semantic continuity between data/information/knowledge and geometric models is still required. The reported works consist in the a posteriori modelling of relationships between data embedded in the stakeholders' models and the parameter-based geometric model created a priori via an implicit interpretation of the stakeholders' design intents [6,7].

The paper then aims at addressing the hypothesis to create a formal semantic continuity between the stakeholders' data models and related geometric models. The paper highlights interface models embedding formal links. Both models are then co-generated by embedding relationships amongst design intents and form features. Considering the concurrent engineering strategy augmented with proactive capabilities, the stakeholders are expected to introduce their knowledge as early as possible in the PDP [8]. The generation of the geometric models is therefore the pure result of the knowledge synthesis process when data is created or modified. Backward design can be also processed to find the rationale of all generated geometric features. To do so, the proposed interface models, are built upon the concepts of flow [9] via geometric skeletons and surfaces $[10,11]$. A flow represents implicitly trajectories, surfaces and volumes as geometric features for generating the detailed parts' CAD model (cf. Fig. 1). The concept of flow seems to be enough generic and suitable to integrate multiple stakeholders' knowledge related to different geometric modelling scales.

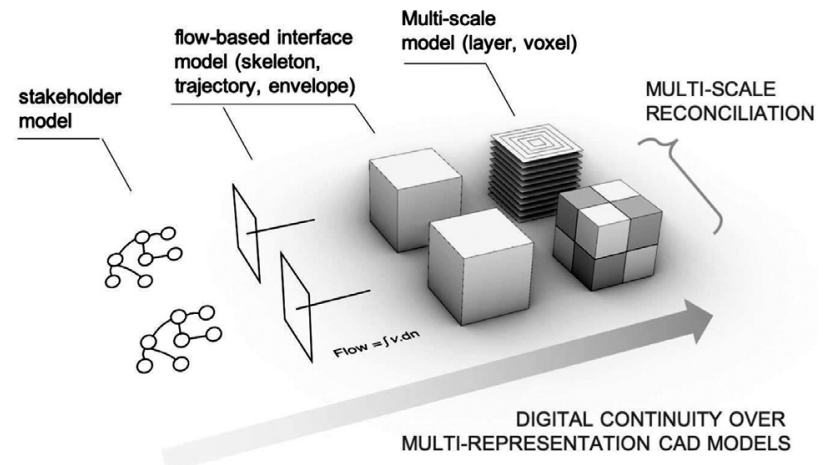

Fig. 1. Theoretical representation of the flow concept.

\subsection{Design project reviews and decision-making based on multi- representations $C A D$ models}

Over the PDP, the digital mock-up is considered as the central shared communication driver, especially during design project reviews. Indeed 3D representation presents a near common sense understanding by all the involved stakeholders. Decision-making is then channelled on a unique geometric model that represents the 'work in progress' solution [11]. However, this central CAD model is in fact a trade-off amongst all geometric intents of every stakeholder. Each one must check and validate or not whether this model is acceptable or ready for each of its expertise [12].

By following the first hypothesis, the paper then proposes to extend the digital semantic continuity with new design review mechanisms based on multiple CAD representations related to each stakeholders' design intents. In such a way, reconciliation mechanisms will ensure the consistency amongst all geometric representations. The advantage of this proposal is to ease the cognitive interpretation of the stakeholders and not to force them to join a unique geometric representation that is not always enough aligned to their expertise (i.e. design intents).

\section{Flow-based interface models concept}

\subsection{Theoretical flow concepts}

As presented in Fig. 1, the notion of flow can be defined as any variable travelling through surface(s) (i.e. skin) by following a given trajectory (i.e. skeleton). The flow of such variable is considered as the integration over the entire trajectory of the scalar product between the vector of the variable and the trajectory element (see Eq. (1)).

Flow $=\int \vec{v} \cdot d \vec{n}$

Explicitly, a flow embeds the following geometric elements:

- A continuous linear element (i.e. skeleton) representing the trajectory of the flow.

- Transverse surface elements (i.e. skins) representing the set of surfaces crossed by the variable. The crossed surface element can indeed evolve (in terms of topology and dimensions) along the trajectory.

- A volume generated by the integration of transverse surface elements on the trajectory.

- An envelope surface (i.e. skin) representing the external envelope of the generated volume.

Therefore, the main objective dealt in the following sections is to propose stereotypes of these flow elements for each of the stakeholders' expertise involved in the PDP.

\subsection{Functional modelling based on energy flows}

In the PDP, the initial stage considers requirements specifications and functional analysis. At this stage, design methods are theoretically supposed to specify functional surfaces (as-required) that have to be independent of any technological solution (i.e. manufacturing processes, assembly processes, etc.). To rationale the functional surfaces, a first stereotype of the flow-based skin and skeleton model is proposed to support the semantic continuity amongst requirements, functional analysis and form features. The originality of the work consists in enhancing efforts initiated in $[9,13,14]$ around the concept of energy flow and bond graph modelling. By considering systems as generating, transforming or consuming energy, the conceptual modelling principles are as follows:

- The functions of the system - as conventionally defined in functional analysis approaches - are associated with a physical principle allowing them to be carried out.

- Each physical principle is associated to an energy flow. Bond graphs are used to represent multi-energy flow with its generalised variables (i.e. effort and current), the type of flow (e.g. mechanical, electrical, magnetic, etc.) and the type of flow circulation/junction (i.e. conduction, insulation, semi-conduction).

The energy flow makes possible to bring out a certain number of geometric elements relating to the functional modelling of the system and its associated physical principles:

- Functional surfaces are the surfaces of each component of the system through which an energy flow circulates.

- The flow trajectory represents the energy field lines (e.g. effort) supported by one or more components.

Fig. 2 describes energy flow modelling through a vice system as illustrative case. The main function of the system is to transform the rotational flow in a translation one.

At this stage, the topology of the geometric elements (i.e. trajectory and surfaces) is not known yet. This confirms the "least commitment' approach based on geometric multi-representations. Other stakeholders will enhance the geometric definition of the system by integrating (synthesis) their design intents.

\subsection{Assembly modelling based on kinematic flows}

This assembly knowledge synthesis is based on parts' kinematic flows. It is therefore the second stereotype of flow-based model to address assembly issues in the early design stages. This conceptual model works with a preliminary bill of materials (BOM) highlighting the main categories of moving components. Afterwards, the idea 

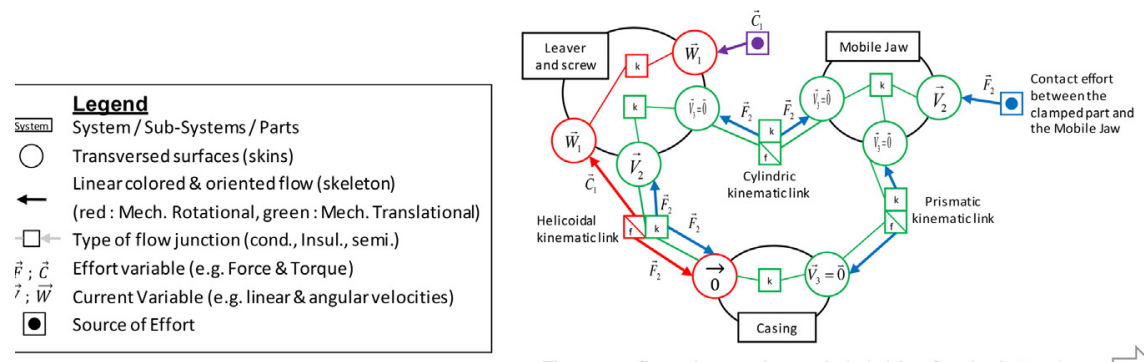

Energy flow-based model (skin \& skeleton)

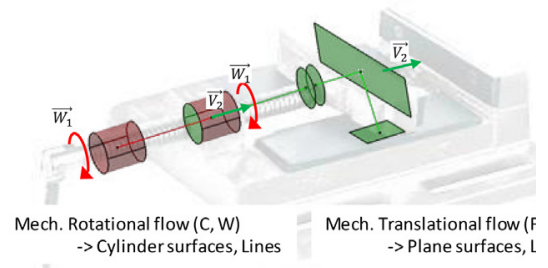

As-required 1D \& 2D CAD model

Fig. 2. Energy flow-based modelling for semantic continuity toward as-required geometric representation. (For interpretation of the references to colour in this figure legend, the reader is referred to the web version of this article.)

consists in detailing all the components of the system in order to map all physical part-to-part relationships. Such additional relationships provide information about the nature of the physical contacts in terms of kinematic pairs and/or technological pairs [11], and gives the opportunity to the assembly planner to introduce assembly logical order for each physical constraint. As a result, on the one hand, the nature of physical pairs will introduce assembly skeletons needed to construct interface skeletons and later on functional surfaces, and on the other hand, precedence constraints from the assembly planner will impact the product structure in order to get a manufacturing BOM via the preliminary generated assembly sequence. Fig. 3 presents the workflow to construct the design space, on which the final (as-planned) geometry of the system can emerge. To do so, the appropriate intermediary objects (i.e. assembly skeletons, interface skeletons, functional surfaces, and envelope-volumes) require to be aligned with functional energy flows as well a manufacturing constraints and knowledge. In other words, reconciliation mechanisms are still needed to connect as-required, as-planned and as-manufactured geometric skeletons to ensure an entire knowledge synthesis. In addition to this forward workflow towards manufacturing - covering multi-representation of assembly design and planning intents - one can identify a backward one, as assembly and manufacturing are two interrelated domains of expertise. Indeed decisions on manufacturing technologies can also affect assembly aspect of the system at different scales. As an example, emerging additive manufacturing techniques have given the opportunity to fabricate complex parts in a layer-bylayer manner but also to build multi-material parts. This means addressing the material distribution within the geometry with an assembly point of view, where network of parts need to be defined at smaller scales to include the relationships of materials. In such a context, the geometry defined at the macro-scale can be broken down into multiple voxels at a lower scale (as illustrated in Fig. 1). Consequently, this backward workflow will require reconciliation means of the geometric representations over the different scales.

\subsection{Manufacturing BOM modelling based on material flows}

After the conceptual and embodiment design stages, the paper addresses the detailed design phase through the Design For Manufacturing (DFM) activity. Once again, a flow-based model is stereotyped to create the semantic continuity between manufacturing knowledge (m-BOM) and as-manufactured form features. Here the interface model represents the material flow generated by each manufacturing operation. The envelope-volume of the flow corresponds to the material volume operated by the manufacturing operation. Therefore, a volume can be added (e.g. casting process), removed (e.g. cutting process) or deformed (e.g. forging or stamping processes). For manufacturing representation, since manufacturing knowledge is considered at each scale, the flow should also be seen at different scales to support the knowledge synthesis (Fig. 4):

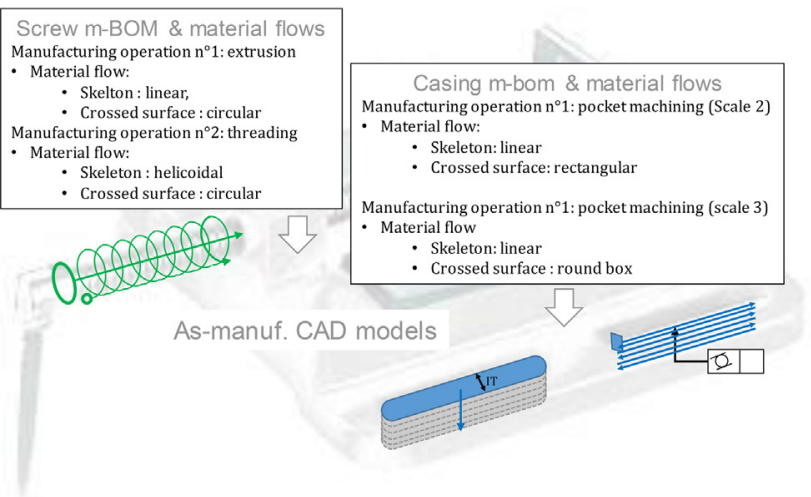

Fig. 4. Manufacturing BOM modelling toward as-manufactured multi-scale geometric representation. (For interpretation of the references to colour in this figure legend, the reader is referred to the web version of this article.)

- Scale 1 corresponds to the material flow representing the interaction between tool and material (e.g., melting point in a fused deposition modelling technique or cutting point for machining).

- Scale 2 stands for the 1D tool trajectory level (i.e. tool path and ripple tolerances).

- Scale 3 is the mesoscopic level that represents the set of trajectories seen as a 2,5D section (e.g., dimensional tolerances of the end mill in cutting process).

- Scale 4 represents the macroscopic overview (e.g., draft constraint in casting process) of the part.

In that DFM step, a reconciliation mechanism is used to ensure the consistency of each model at each scale with respect to the other ones. Homogenisation mechanism actually allows the co-generation of CAD models from scales $n$ to $n+1$.
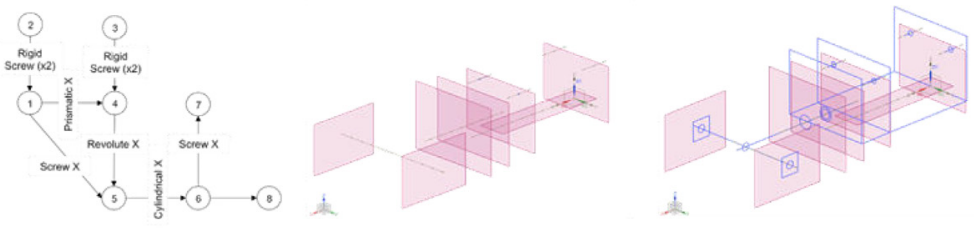

Assembly
skeletons

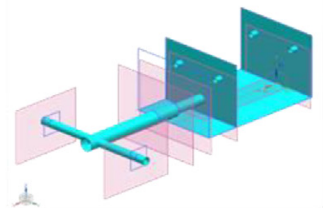

Interface

skeletons

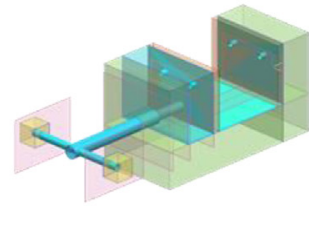

Envelopesvolumes

Fig. 3. Top-down definition of design assembly skeletons toward as-planned geometric representation. 


\subsection{Reconciliation mechanisms for multiple CAD models}

By introducing interface models to manage the emergence of geometric models via the concurrent integration of multiple stakeholder knowledge, multiple BOMs have to be managed with explicit relationships. The latter can be the support of reconciliation mechanisms for ensuring propagation and semantic continuity [15]. Beyond these relationships, the founding hypothesis of these works, for which several CAD representations are generated (Figs. 2, 3 and 4), requires new reconciliation mechanisms as follows:

- Verification of the topological consistency of all 1D, 2D and 3D CAD features since they are not propagated from the same interface models. For example, the as-manufactured 3D form features should be consistent with the as-required 1D and 2D ones.

- Interoperability mechanisms between CAD representations so that each one can be considered as an entry for another stakeholder.

- Decomposition or homogenisation. The most illustrative example is the as-manufactured CAD that represents different geometric scales (i.e. macro, meso and micro) depending on the knowledge to be integrated. Form features homogenisation should therefore be analogue to material characteristics homogenisation [16].

\section{Conclusion, validation and future work}

As conclusion, the proposed work has showed how the generic concept of flow is used to formalize interface models supporting the semantic continuity between stakeholders' design intents and geometric features. The first hypothesis related to semantic continuity is illustrated for three of the PDP stakeholders. The related geometric elements are therefore the pure result of the concurrent knowledge integration made by the stakeholders involved in the PDP. The results also illustrates that each design intent lets emerge different 1D, 2D or 3D form features. The second hypothesis is then confirmed; reconciliation mechanisms should be developed to let the co-generation of each CAD model. The design review will be enhanced instead of having a unique $C A D$ model created with non-rationale trade-offs.

In terms of validation, the results of this paper show the gains for the scientific and industrial communities since it goes back to the fundamentals of the design process and the creation of the CAD model. The techno-pushed approaches more or less imposed by the evolutions of CAD systems have hidden that the PDP is an activity made of decisions centred on expertise and not only focused on the definition of a geometric model. The proposal therefore provides an important functionality to support the design process and ensure both semantic interoperability and digital continuity from design intents to form features. Another gain concerns product development that has become 'justified', and for which there is a digital continuity supporting any changes related to review/rethink past decisions over systems' lifecycle. In that sense, the design process becomes agile and the design solutions space can evolve accordingly with changes of requirements, technologies, manufacturing processes, assembly processes and so on.

To go forward with the validation process, current research work is focused on the definition of a formal framework to assess the performance of the proposal. Such framework is still lacking in the scientific communities. By revising the PDP and its dedicated product model, the proposal aims at ensuring the following drivers:

- The capacity of tracking and retrieve design intents and design rational

- The capacity to support what-if analyses

- The capacity to model design solutions space to support innovation

The framework will then propose some benchmarks in order to measure those characteristics and formally compare with other solutions of the scientific and industrial community.

Future work will focus on the validation of the second hypothesis via a cognitive study on the interest of sharing a single CAD model during the decision-making phases versus sharing several CAD models from stakeholders. Another work will address the enrichment and the development of the multi-BOM modelling PLM environment.

\section{References}

[1] Chandrasegaran SK, Ramani K, Sriram RD, Horvath I, Bernard A, Harik RF, Gao W (2012) The evolution, challenges, and future of knowledge representation in product design systems. Computer-Aided Design 45(2):204-228.

[2] Roucoules L, Tichkiewitch S (2015) Knowledge synthesis by least commitment for product design. CIRP Annals 64(1):141-144

[3] Lutters E, van Houten FJAM, Bernard A, Mermoz E, Schutte CSL (2014) Tools and techniques for product design. CIRP Annals 63(2):607-630.

[4] Groche P, Schmitt W, Bohn A, Gramlich S, Ulbrich S, Günther U (2012) Integration of manufacturing-induced properties in product design. CIRP Annals 61(1):163166.

[5] Molcho G, Zipori Y, Schneor R, Rosen O, Goldstein D, Shpitalni M (2008) Computer aided manufacturability analysis: Closing the knowledge gap between the designer and the manufacturer. CIRP Annals 57(1):153-158.

[6] Skarka W (2007) Application of MOKA methodology in generative model creation using CATIA. Engineering Applications of Artificial Intelligence, Soft Computing Applications 20:677-690.

[7] Demoly F, Roth S (2017) Knowledge-based parametric CAD models of configurable biomechanical structures using geometric skeletons. Computers in Industry 92-93:104-117

[8] Ballu A, Falgarone H, Chevassus N, Mathieu L (2006) A new design method based on functions and tolerance specifications for product modelling. CIRP Annals 55 (1):139-142

[9] Hirtz, Stone RB, McAdam DA, Szykman S, Wood KL (2002) A functional basis for engineering design: Reconciling and evolving previous efforts. Research in Engineering Design 13(2):65-82.

[10] Skander A, Roucoules L, Klein Meyer JS (2008) Design and manufacturing interface modelling for manufacturing processes selection and knowledge synthesis in design. International Journal of Advanced Manufacturing Technology 37(5-6):443454.

[11] Demoly F, Toussaint L, Eynard B, Kiritsis D, Gomes S (2011) Geometric skeleton computation enabling concurrent product engineering and assembly sequence planning. Computer-Aided Design 43(12):1654-1673.

[12] Kondoh S, Tezuka A (2014) Synthesis of verification models in multidisciplinary design of complex engineered systems. CIRP Annals 63(1):145-148.

[13] Ullman D (2009) The Mechanical Design Process, 4 ed. McGraw-Hill Education.

[14] Albers A, Matthiesen S, Ohmer M (2004) Evaluation of the element model "working surface pairs \& channel and support structures". in Tichkiewitch S, Brissaud D, (Eds.) Methods and Tools for Co-operative and Integrated Design, Springer, Dordrecht, 353-362.

[15] Fenves S.J. (2002) A core product model for representing design information. NIST Internal Report- 673.

[16] Liu X, Shapiro V (2016) Homogenization of material properties in additively manufactured structures. Computer-Aided Design 78:71-82. 